\title{
Compact Combline Filter Embedded in a Bed of Nails
}

\author{
Mariano Baquero-Escudero, Member, IEEE, Alejandro Valero-Nogueira, Senior Member, IEEE, \\ Miguel Ferrando-Rocher, Student Member, IEEE, Bernardo Bernardo-Clemente, \\ and Vicente E. Boria-Esbert, Fellow, IEEE,
}

\begin{abstract}
In this paper, we propose a compact topology for high frequency bandpass filters using coaxial cavities embedded in a bed of nails, including a complete design procedure combining equivalent circuit models and full-wave simulators. The resonance generated around a shortened cylindrical nail of the bed hosting structure is used as the basic element of the proposed filter, which is fed through Groove Gap Waveguides. For design purposes, an equivalent circuit model of the considered resonance is first obtained, and then the coupling levels between resonators are recovered with the distance between adjacent shortened nails. In order to validate the proposed structure and its design procedure, a filter prototype with a bandpass response (centered at 30 $\mathrm{GHz}$ and with relative bandwidth of $1.7 \%$ ) has been designed, manufactured and measured. Good experimental results, in terms of insertion losses (with a minimum value of $1.6 \mathrm{~dB}$ ) and return losses (greater than $16.6 \mathrm{~dB}$ in the whole pass-band), have been achieved.
\end{abstract}

Index Terms-Aggressive space mapping (ASM), band-pass filter, combline filter, groove gap waveguide, periodic structures, stopband response.

\section{INTRODUCTION}

$\mathbf{I}$ $\mathrm{N}$ recent years, periodic structures have regained considerable relevance in the design of passive microwave devices. The frequency selective properties of these periodic structures can be used for many different purposes, [1]. For instance, artificial magnetic conductor (AMC) is one of that non-physical concepts that can be physically realized thanks to periodic structures. The combination of two parallel plates, one being an AMC and the other a perfect electric conductor (PEC), was proposed as a platform providing a stopband response, in which different circuits can be embedded in a contactless fashion [2], [3]. The analysis and modeling of these structures, recently named gap waveguides, can be found in [4], [5].

The practical usage of this technology has been growing in the last years, as confirmed by the increasing number of designed transitions between gap waveguides and other classical

This work was supported in part by the Spanish Government, Ministry of Economy, National Program of Research, Development and Innovation through the project SATCOM-KA "New antennas for mobile satellite communications in Ka band" under Project TEC2016-79700-C2-1-R and "Demostradores tecnologicos de filtros y multiplexores con respuestas selectivas y sintonizables en nuevas guias compactas para aplicaciones espaciales" under Project TEC2016-75934-C4-1-R. (Corresponding author: Mariano BaqueroEscudero.)

The authors are with the Instituto de Telecomunicaciones y Aplicaciones Multimedia (iTEAM) Universitat Politècnica de València, 46022-Valencia, Spain e-mail: (see http://www.iteam.upv.es/user.php)

Manuscript received July 25, 2018; revised September 9, 2018; accepted January 12, 2019 technologies (see [6]-[11]), and their practical employment for implementing many other passive components. Among them, we can find power dividers, [11], antenna feeding network, [12], and different filter topologies for microwave and millimeter-wave applications, [13]-[19].

More recently, new topologies for slot array antennas with enhanced radiation properties, based on a resonance around a shortened nail of the gap waveguide structure, have been successfully proposed in [20]-[23]. As it was identified in [21], such resonance is very similar to the one of a coaxial resonant cavity, which are typically employed in combline waveguide filters [24], [25]. Therefore, this resonance could be used for implementing a compact version of combline filters in gap waveguide technology.

In this paper, we demonstrate the practical implementation of a combline filter seamlessly integrated in a supporting bed of nails, making use of coupled resonances due to shortened nails of the hosting structure. For designing these filters, an efficient procedure based on the aggressive space mapping technique, [26], used in combination with a classical method based on coupled-resonator circuits [27], has been proposed and illustrated with a practical case. For this purpose, a bandpass response centered at $30 \mathrm{GHz}$ and with a bandwidth of $500 \mathrm{MHz}$ (i.e., 1.7\%), in-band return losses of $20 \mathrm{~dB}$ and ripple level of $0.0436 \mathrm{~dB}$, has been considered.

The paper is organized as follows. In section II, we have studied the stopband behaviour of the periodic structure used for embedding the proposed filters. Section III includes a detailed analysis of the coaxial cavity resonance, providing an equivalent circuit for its accurate modeling. Then, Section IV details the complete design procedure of the proposed coaxial filter based on the ASM technique. Its practical realization, including experimental validation results, are shown in Section V. Finally, Section VI outlines the main conclusions of this work.

\section{STOPBAND OF THE BED OF NAILS}

A periodic structure provides a stopband frequency response that can be used for different purposes. Several studies of the stopband properties of gap waveguides have been already published, as can be seen in [28], [29]. Due to its simple machining, a square section nail (see Fig. 1) is frequently used as the unit cell of periodic structures, being able to provide a stopband response with an octave bandwidth. The relationship between the provided stopband response and the geometrical 


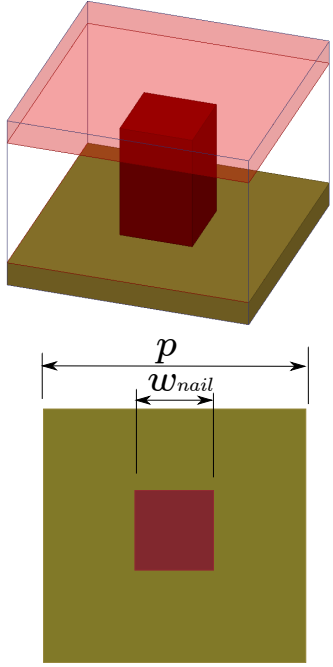

Fig. 1. Geometry of a square section nail used in periodic structures.

dimensions of the unit cell, i.e., $w_{\text {nail }}, h_{\text {nail }}, h_{\text {air }}$ and $p$ in Fig. 1, has been studied with detail in [2] and [29].

One of the main advantages of gap waveguide technology, lies in the fact that perfect shielding is not required to keep electromagnetic fields confined in the waveguide. Conventional gap waveguides leave a small air gap (less than $\lambda / 4)$ between the nails top and the lid. The high-impedance condition created by the bed of nails forces a stopband in the air gap. However it has been observed [30] that in fact the presence of the air gap is not strictly necessary and the lid can rest directly onto the nails. Therefore some nails may be in contact with the nails while others may not, due to machining imperfections, but the stopband condition is still retained or even increased in frequency range. Such arrangement has been named zero-gap and it provides certain practical advantages since assuring a thin air gap is not an easy task, particularly in the millimeter-wave band and above.

We have performed a parametric analysis in terms of the variable $h_{\text {air }}$, considering values for $h_{\text {air }}$ between 0 and 100 microns, as well as keeping constant the sum of the nail height, $h_{\text {nail }}$, and the air gap, $h_{\text {air }}$, (i.e., $h_{\text {air }}+h_{\text {nail }}=1.8 \mathrm{~mm}$ ). For the other dimensions of the unit cell shown in Fig. 1, we have chosen $w_{\text {nail }}=0.9 \mathrm{~mm}$ and $p=2.5 \mathrm{~mm}$, which provides a stopband response of enough bandwidth, so that it will be possible to allocate the bandpass response of the coaxial filter to be designed.

In Fig. 2, we show results obtained with HFSS eigenmode solver (from $A N S Y S$ ), where we can see that the minimum frequency of the stopband response tends to $0 \mathrm{GHz}$ when the air gap value is progressively reduced up to be null. On the other hand, the maximum frequency of the stopband response is practically kept constant for different values of $h_{\text {air }}$, around $70 \mathrm{GHz}$.

From this study, it can be seen that the stopband bandwidth of these periodic structures tends to grow when the air gap decreases, and that the stopband response is preserved (in terms of bandwidth) even for a zero-gap case.

For hosting the bandpass coaxial filter proposed in this

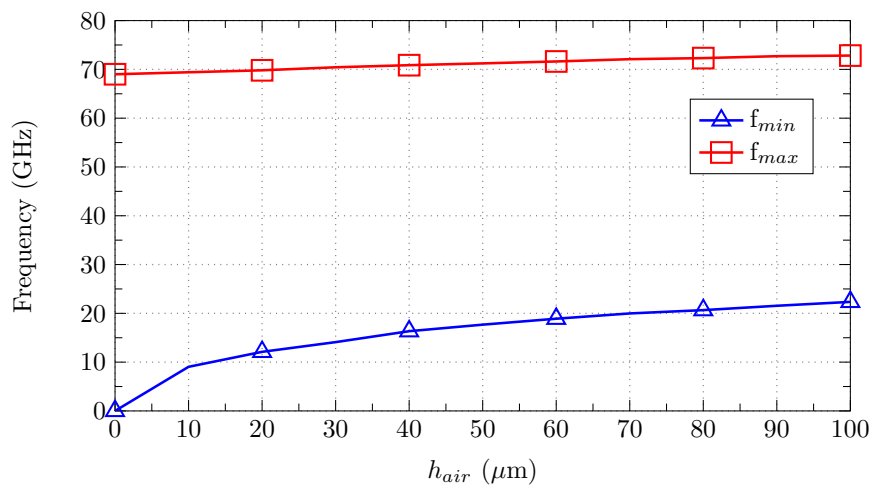

Fig. 2. Stopband behaviour versus $h_{\text {air }}$, with $w_{\text {nail }}=0.9 \mathrm{~mm}, h_{\text {nail }}+$ $h_{\text {air }}=1.8 \mathrm{~mm}$ and $p=2.5 \mathrm{~mm}$.

work, we will make use of zero-gap to alleviate fabrication burden.

\section{Shortened NAIL Resonance Modeling}

As can be seen in Fig. 3 (results from ANSYS HFSS eigenmode solver), a shortened nail embedded in a bed of square section nails has a clear resonant behaviour around it. It can be observed that the electric field is vertical at the air gap and radial near the base of the resonator. From these results (and the resonator topology), it can be concluded that this resonance is similar to the fundamental one of a coaxial resonant cavity used in more classical combline waveguide filters [24]. Compared with the resonators made in RGW and GGW presented in [16] the main advantage of the proposed combline resonator is related to its smaller size, that provides a cleaner out-of-band frequency spectrum response.

It can be noticed that the shortened nail has been chosen with cylindrical shape. This shape has been selected to avoid square corners, which can concentrate intense currents which normally imply higher losses. Other advantage of using cylindrical shapes is related to its meshing for modeling purposes, because with curved surfaces and using a curvilinear meshing, the number of requested tetrahedrons is lower and the computation effort is substantially reduced.

Additionally, as it also happens with an equivalent coaxial waveguide resonator, it has been checked that the resonance frequency of the proposed structure (Fig. 3) can be effectively controlled with the height of the cylindrical nail (or equivalently, with the value of the gap between the shortened nail and the top metal piece).

Therefore, in order to model the resonance around the shortened nail, it seems reasonable to choose the equivalent circuit shown in Fig. 4, which is composed of a length of shortcircuited coaxial transmission line (representing the cylindrical nail connected to the bed metallic base), loaded at its end with a capacitor (modeling the gap effect between the nail and the top metal plate)

As it is well known, the resonance condition of the proposed equivalent circuit (Fig. 4) is given by:

$$
Y_{L}+Y_{R}=0
$$



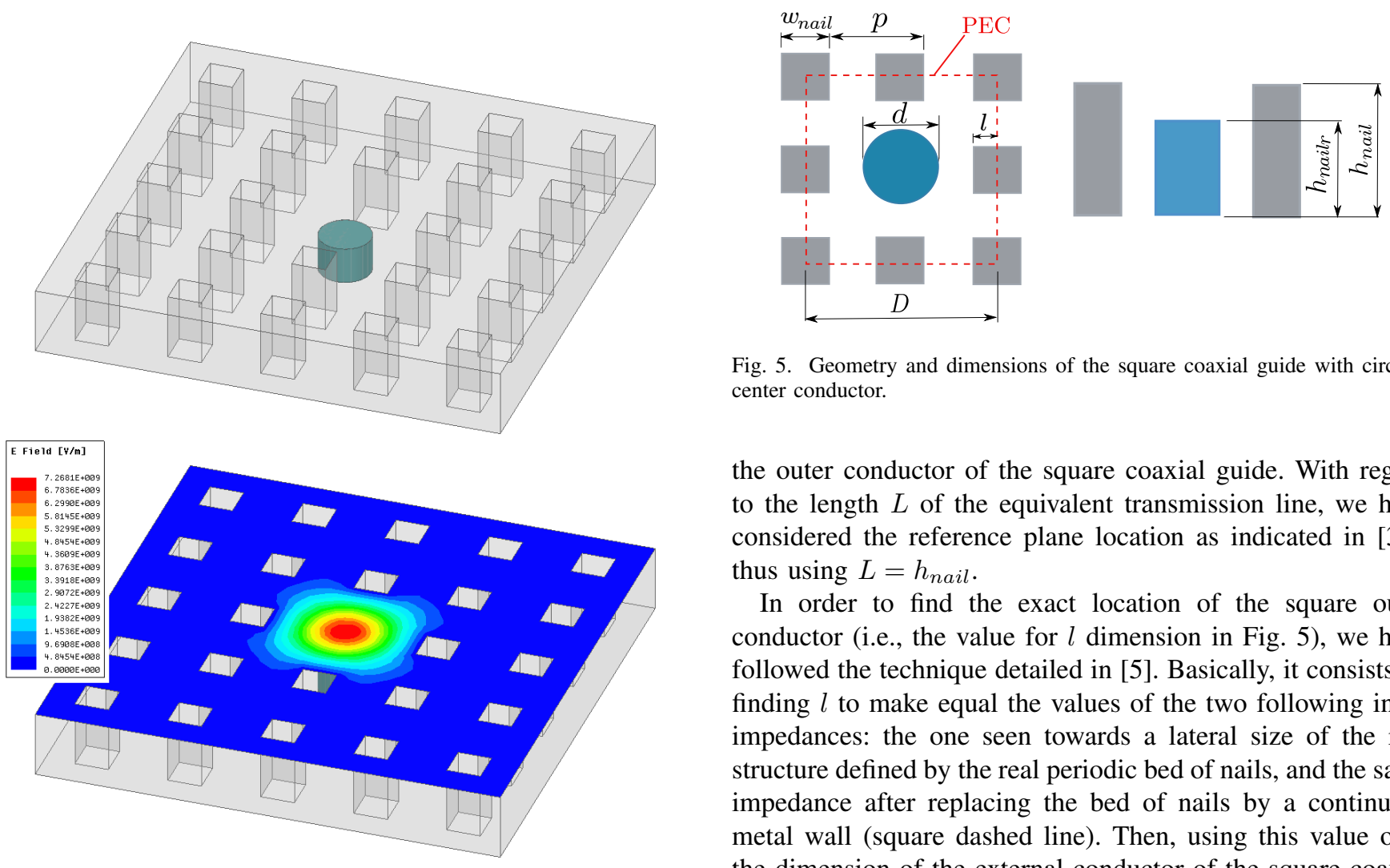

Fig. 5. Geometry and dimensions of the square coaxial guide with circular center conductor.

the outer conductor of the square coaxial guide. With regard to the length $L$ of the equivalent transmission line, we have considered the reference plane location as indicated in [31], thus using $L=h_{\text {nail }}$.

In order to find the exact location of the square outer conductor (i.e., the value for $l$ dimension in Fig. 5), we have followed the technique detailed in [5]. Basically, it consists on finding $l$ to make equal the values of the two following input impedances: the one seen towards a lateral size of the real structure defined by the real periodic bed of nails, and the same impedance after replacing the bed of nails by a continuous metal wall (square dashed line). Then, using this value of $l$, the dimension of the external conductor of the square coaxial guide (i.e., $D$ ) is obtained through the following expression:

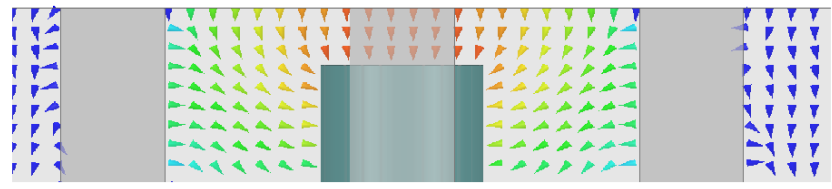

Fig. 3. Geometry of a cylindrical shortened nail within a bed of square nails, and electromagnetic field distribution at the corresponding resonant frequency.

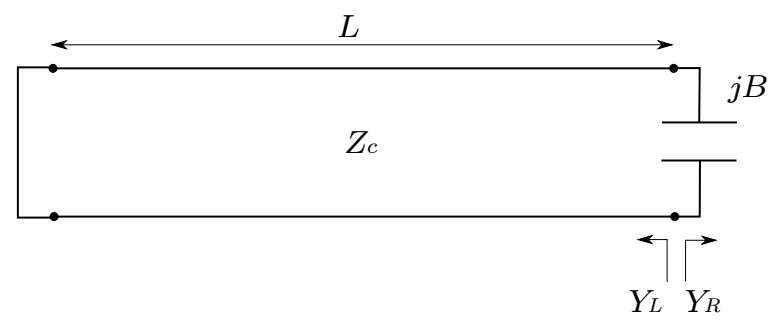

Fig. 4. Equivalent circuit model of the considered resonance around the shortened nail.

being:

$$
\begin{gathered}
Y_{L}=-j Y_{c} \frac{1}{\tan \beta L} \\
Y_{R}=j B=j \omega C
\end{gathered}
$$

where $Y_{c}$ is the characteristic admittance of the transmission line representing the cylindrical nail. In order to compute $Y_{c}$, an equivalent square coaxial guide with circular center conductor is proposed. In Fig. 5 we can see all involved geometrical variables, as well as dashed red lines defining

In our case, considering again $w_{\text {nail }}=0.9 \mathrm{~mm}, h_{\text {nail }}=1.8 \mathrm{~mm}$ and $p=2.5 \mathrm{~mm}$, we obtain (after applying the previously cited technique), a value of $l=0.181 \mathrm{~mm}$, and therefore $D=4.462$ $\mathrm{mm}$. Choosing a diameter for the shortened cylindrical nail of $d=1.4 \mathrm{~mm}$, we compute the value for the characteristic impedance of the square coaxial guide as follows [32]:

$$
Z_{c}=60 \ln \left(\frac{1.0787 D}{d}\right)=74.09 \Omega
$$

To calculate the value for the capacitor of the considered equivalent circuit, the following expression (proposed by Marcuvitz in [31] for modeling the termination of a coaxial line by a capacitive gap) can be used:

$$
\begin{aligned}
\frac{B}{Y_{c}}=\left(\frac{2 d}{\lambda_{0}} \ln \frac{D}{d}\right)\left[\frac{\pi d}{8\left(h_{\text {nail }}-h_{\text {nailr }}\right)}+\right. \\
\left.\ln \left(\frac{D-d}{2\left(h_{\text {nail }}-h_{\text {nailr }}\right)}\right)\right]
\end{aligned}
$$

However, as explained in [31], the previous expression corresponds to the case of a cylindrical coaxial line (i.e., with a circular outer conductor), and it is only valid for a certain range of geometrical dimensions. Therefore, in order to obtain more accurate results for the normalized susceptance of the capacitor, it will be necessary to use full-wave electromagnetic (EM) simulators considering the real geometry of our equivalent coaxial line. For this purpose, we have again employed the commercial tool ANSYS HFSS. 


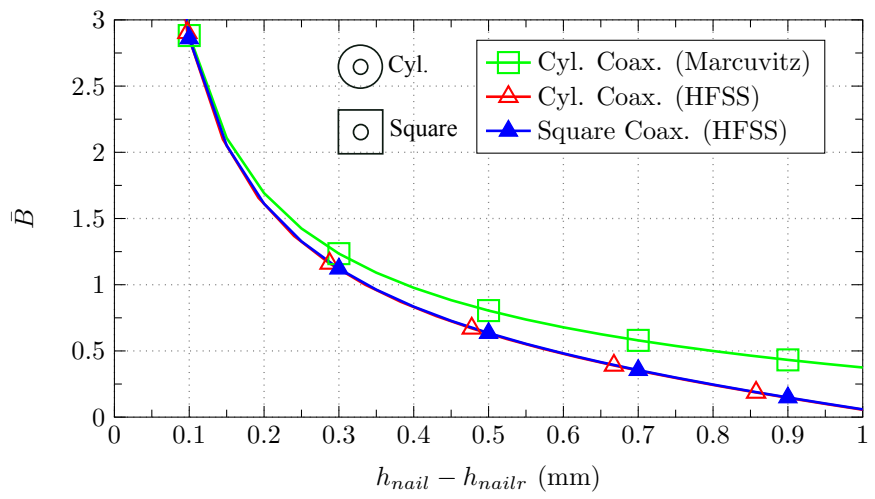

Fig. 6. Normalized equivalent susceptance $(\bar{B})$ versus the gap height (i.e., $\left.h_{\text {nail }}-h_{\text {nailr }}\right)$.

For the same shortened nail configuration studied before, we have computed the normalized susceptance values for different gap heights (or $h_{\text {nail }}-h_{\text {nailr }}$ ) at a frequency of $30 \mathrm{GHz}$, employing the Marcuvitz expression in (5) and ANSYS HFSS commercial software (considering both a circular and a square outer conductor for equivalent coaxial guide). All these results are compared in Fig. 6, where it can be concluded that ANSYS HFSS results are independent of the shape (circular or square) selected for the outer conductor of the equivalent coaxial guide, and that expression (5) provides valid results for small values of the gap height.

Once all the element values of the proposed equivalent circuit (see Fig. 4) have been computed, the resonance frequency of the considered resonator can be found solving the transcendental equation defined in (1)-(2), using any numerical method suitable for this purpose. This procedure has been applied to the very same resonator geometry under study, and the resonance frequencies obtained for different values of the shortened nail height $\left(h_{\text {nailr }}\right)$ are shown in Fig. 7 , where they are successfully compared with the results from ANSYS HFSS eigenmode solver.

From the results displayed in Fig. 7, two main conclusions can be outlined. The first one is that the proposed equivalent circuit is really modeling the resonant behaviour of the selected coaxial resonators very accurately, and, therefore, it can be effectively used for accelerating the design process of the corresponding shortened nail topology. Secondly, the curves relating the resonance frequency with the height of the shortened nail can be very well approximated (as can be seen in Fig. 7) by means of a linear equation, with a slope of $m=-16.4 \mathrm{GHz} / \mathrm{mm}$ for the particular example considered in such figure. This linear behaviour will be also employed, as explained next, in the design procedure outlined for the proposed combline filters in gap waveguide technology.

\section{Filter Design Procedure}

In this section, we will present the physical topology of the proposed coaxial filters, based on the cascaded coupling of the resonances due to shortened nails that have just been properly modeled. The complete design procedure of these filters, based on a very efficient approach combining circuital and

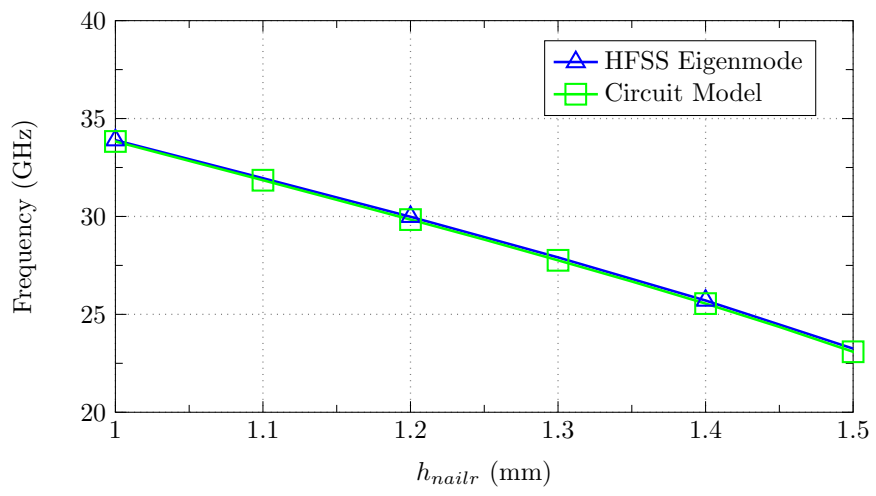

Fig. 7. Resonance frequency versus $h_{\text {nailr }}$ (height of the cylindrical shorted nail), with $w_{n a i l}=0.9 \mathrm{~mm}, h_{n a i l}=1.8 \mathrm{~mm}, p=2.5 \mathrm{~mm}$, and $d=1.4 \mathrm{~mm}$.

electromagnetic simulation models, will be also thoroughly described next.

In order to illustrate the proposed filter topology, and its related design process, we will focus on recovering a bandpass response of $500 \mathrm{MHz}$ centered at $30 \mathrm{GHz}$ (i.e., with a relative bandwidth of $1.7 \%$ ), with in-band return loss and equi-ripple levels of $20 \mathrm{~dB}$ and $0.0436 \mathrm{~dB}$, respectively. Choosing a fourth-order filter, the normalized values for the corresponding low-pass prototype can be easily obtained from classical textbooks, [33]. Then, using well known expressions from [25], [27], we can compute the values of the coupling coefficients $(k)$ and external quality factor $\left(Q_{e}\right)$ of an equivalent circuit based on coupled resonators, which in our particular example are:

$$
\begin{array}{r}
k_{12}=k_{34}=0.0152 \\
k_{23}=0.0117 \\
Q_{e}=55.88
\end{array}
$$

This equivalent circuit representation of the complete bandpass filter, based on coupled series resonators, is shown in Fig. 8 , where all the input variables needed to compute its electrical response are included. In our particular filter example (of order 4 and considering a symmetrical implementation in two equal halves), the only involved variables are the cited $Q_{e}, k_{12}, k_{23}$, $f_{01}$ and $f_{02}$, being $f_{01}$ the resonance frequency of the first and fourth resonators, and $f_{02}$ the one for the second and third resonators of our symmetric configuration.

Using the values for $Q_{e}, k_{12}$ and $k_{23}$ collected in (6), and choosing $f_{01}=f_{02}=30 \mathrm{GHz}$, we have obtained (using the commercial software $A D S$, from Keysight) the ideal bandpass response shown in Fig. 9, which is the objective to be recovered with the physical topology selected for implementing the combline filter.

In the proposed design procedure, we will obtain a first set of physical values for the filter geometrical variables following the well-known method described in [27]. The practical application of this method requires the previous design of the filter resonators, which in our case are the shortened nails discussed in Section III. Selecting the same hosting bed considered before (whose values are included in the caption of Fig. 7), and using the results of such figure, we have selected a value for 


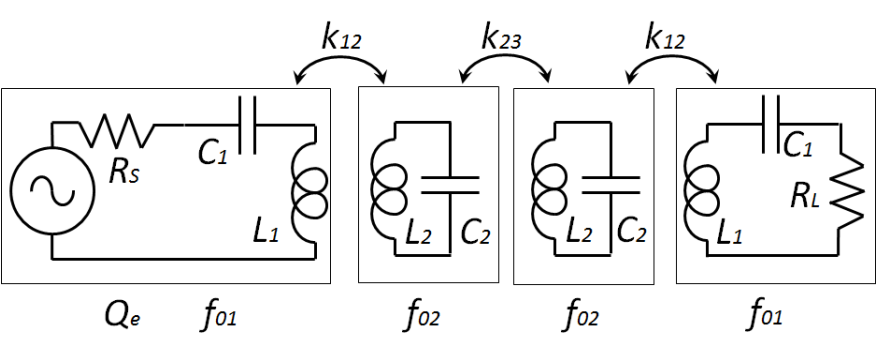

Fig. 8. Equivalent circuit representation of the filter based on series coupled resonators.

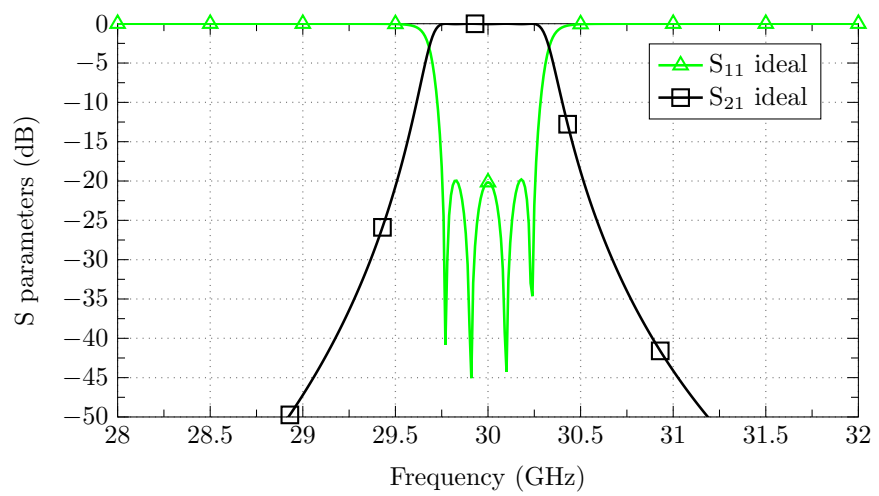

Fig. 9. Ideal band-pass response of the filter equivalent circuit.

$h_{\text {nailr }}=1.2 \mathrm{~mm}$ (which provides, as requested, a resonance frequency equal to $30 \mathrm{GHz}$ ).

Next, we will explain how we can recover, with the appropriate physical structures, the requested values for the other electrical variables (i.e., $Q_{e}, k_{12}$ and $k_{23}$ ) of our equivalent circuit representation.

\section{A. External Quality Factor Implementation}

The input and output cavities of the filter will be fed through two horizontally-polarized groove gap waveguides (GGWs), similar to the ones already employed in [21], that will be properly matched to standard rectangular waveguides WR28 $\left(a_{W R 28}=7.112 \mathrm{~mm}, b_{W R 28}=3.556 \mathrm{~mm}\right)$ used for measurement purposes. Close to the metal base of the resonator the field is radial, thus presenting good matching with the fundamental mode of the GGW used for excitation. A side view of the considered input and output ports can be seen in Fig. 10, where all involved dimensions are included (the values for the hosting bed of square nails are the same ones used before).

As can be seen in Fig. 11, the feeding GGW is ended in a backshort, which is located at a distance, $d_{f}$, from the center of the first resonant shortened nail. This is the physical parameter to be optimized, in order to recover the ideal value for the $Q_{e}$ electrical parameter found before (i.e., $Q_{e}=55.88$ ).

According to [27], the $Q_{e}$ value provided by an input coupling structure (in our case the one of Fig. 11) is evaluated as follows:

$$
Q_{e}=\frac{\omega_{0}}{\Delta \omega_{ \pm 90^{\circ}}}
$$

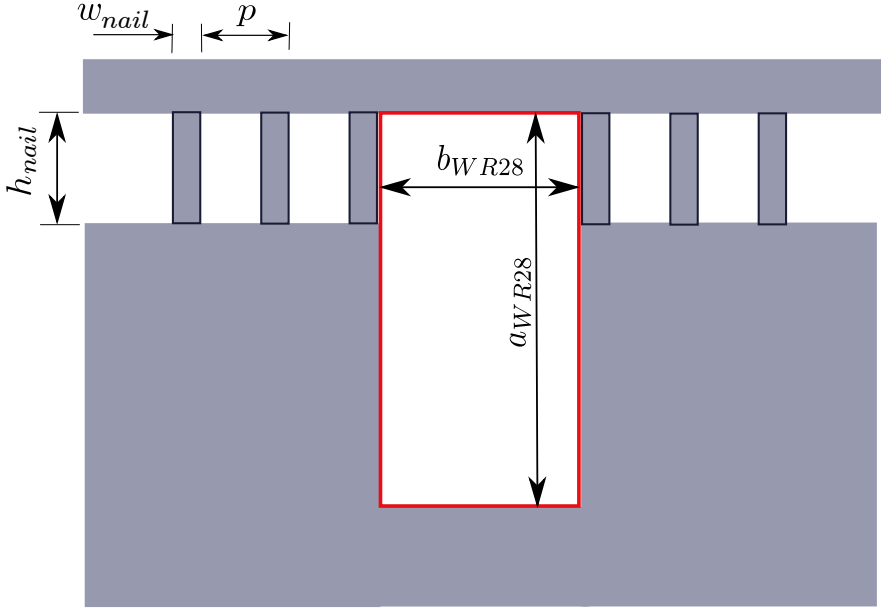

Fig. 10. Side view of an horizontally-polarized GGW feeding the proposed filter.
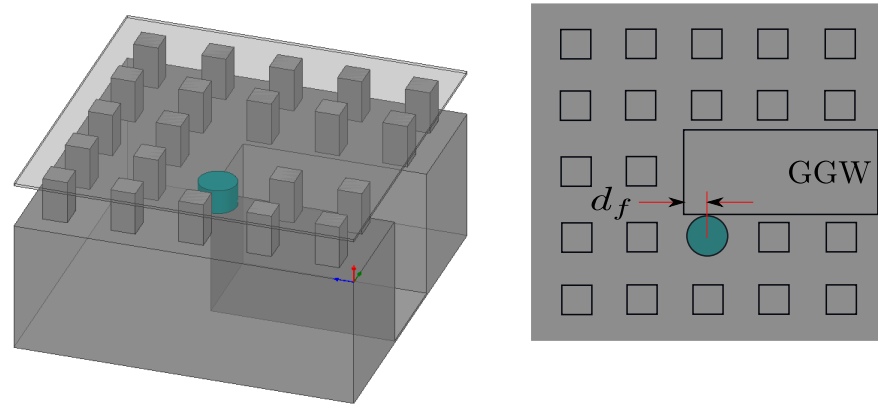

Fig. 11. 3D and 2D views of the input cavity, including the feeding GGW.

where $\omega_{0}$ is the resonance frequency of the considered structure; and $\Delta \omega_{ \pm 90^{\circ}}$ is the difference between the two angular frequencies where the phase of the $S_{11}$ parameter is shifted, respectively, $\pm 90^{\circ}$ with respect to the phase value at $\omega_{0}$.

As recommended in [27], in order to obtain the exact value of $\omega_{0}$ (due to potential loading effects in the structure of Fig. 11), we will compute the group delay of $S_{11}$ through the expression:

$$
\tau_{S_{11}}(\omega)=-\frac{\partial \phi_{S_{11}}}{\partial \omega}
$$

being $\omega_{0}$ the value of the angular frequency where the group delay is maximum.

For design purposes, we have first obtained (using ANSYS $H F S S$ for the electromagnetic simulations) a curve relating the values of $Q_{e}$ that can be achieved with the structure of Fig. 11 for different values of the distance $d_{f}$. From these results (displayed in Fig. 12), we select an initial value of $d_{f}$ $=1.073 \mathrm{~mm}$ for recovering the requested value of $Q_{e}=55.88$.

Additionally, in order to keep the resonance frequency of the loaded input cavity at $30 \mathrm{GHz}$ (center frequency of the objective bandpass response), we have found a new value for the height of the first shortened nail $\left(h_{\text {nailr }}=1.013 \mathrm{~mm}\right)$. For this purpose, a curve relating the resonant frequency of the considered structure (Fig. 11) in terms of $h_{\text {nailr } 01}$ has been obtained. This new design curve resembles the one included 


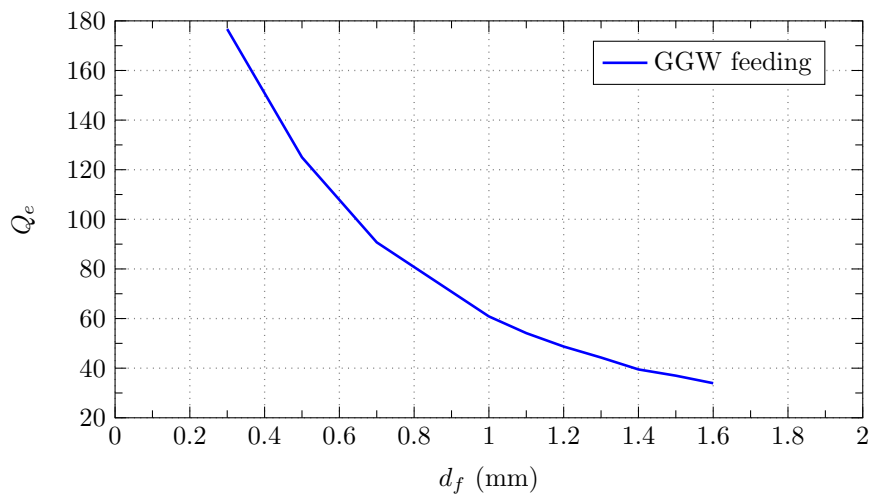

Fig. 12. Qe values versus the physical parameter $d_{f}$ of the input cavity.
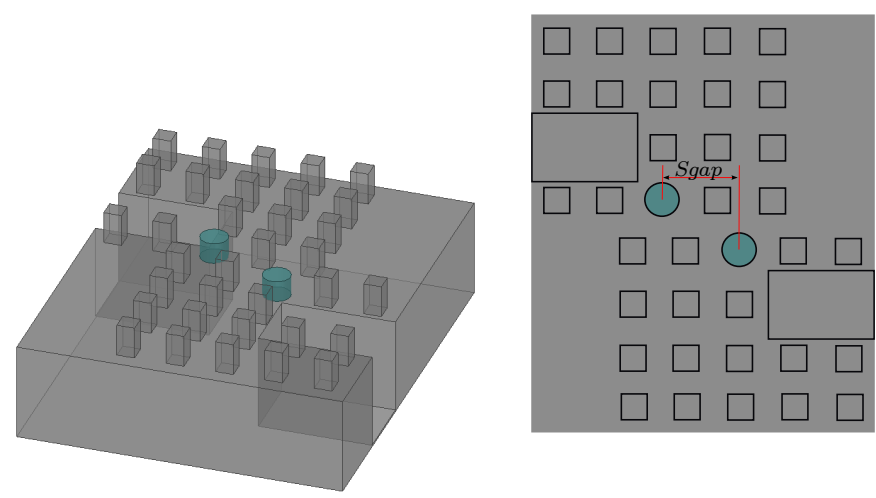

Fig. 13. 3D and 2D views of two coupled adjacent resonators, including weak excitation through GGWs.

in Fig. 7, but it can be now approximated by a linear equation with a slope $m=-15.7 \mathrm{GHz} / \mathrm{mm}$.

\section{B. Coupling Implementation}

In order to implement the coupling between adjacent resonant cavities, the geometrical configuration shown in Fig. 13 is proposed, where the distance, $s_{\text {gap }}$, between the two shortened nails of the resonators can be changed. This physical parameter will be optimized for realizing, in each case, the ideal value of the corresponding coupling coefficient previously computed (i.e., $k_{12}=0.0152$ and $k_{23}=0.0117$ ).

As can be observed in Fig. 13, and with the aim of preserving the density of the bed of square nails surrounding each resonant cylindrical post, the relative distance between consecutive rows of nails is kept constant. Proceeding in this way, the resonant behaviour of each shortened nail will be very practically equal to the one studied in Section III.

For computing the value of the coupling coefficient between the two coupled resonators, as explained in [27], we have weakly excited the structure (in our case with two GGWs as shown in Fig. 13). Then, the two frequencies, $f_{1}$ and $f_{2}$, where the $\left|S_{21}\right|$ of the considered structure has two resonant peaks are identified, and used in the following expression for computing the value of $k$ :

$$
k=\frac{f_{2}^{2}-f_{1}^{2}}{f_{2}^{2}+f_{1}^{2}}
$$

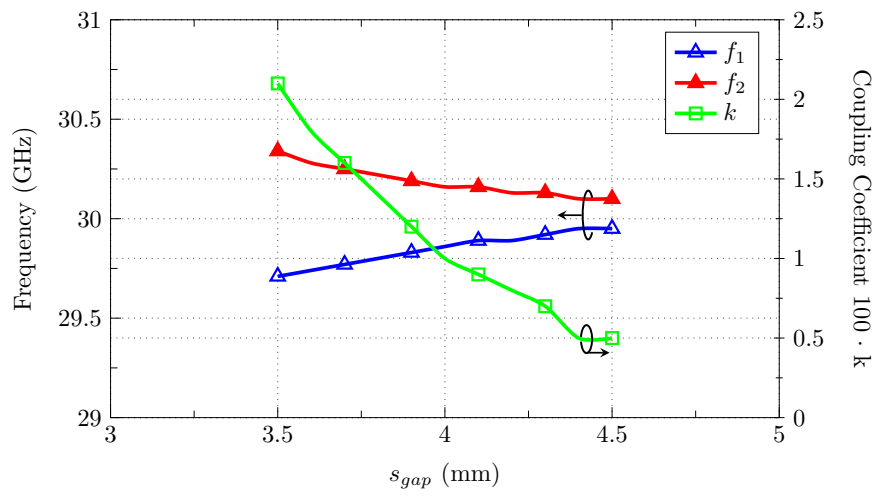

Fig. 14. Values for the frequencies $f_{1}$ and $f_{2}$ used in (9), and the corresponding $k$ values, versus the physical distance $s_{g a p}$ between adjacent shortened nails.

TABLE I

VALUes for ALl Filter DESIGN VARIABles

\begin{tabular}{|c|c||c|c|c|}
\hline Elec. Par. & Opt. Value & Phys. Par. & Init. Value & Final Value \\
\hline \hline$Q_{e}$ & 55.88 & $d_{f}(\mathrm{~mm})$ & 1.073 & 1.002 \\
\hline$k_{12}$ & 0.0152 & $s_{\text {gap } 12}(\mathrm{~mm})$ & 3.715 & 3.743 \\
\hline$k_{23}$ & 0.0117 & $s_{\text {gap } 23}(\mathrm{~mm})$ & 3.892 & 3.942 \\
\hline$f_{01}(\mathrm{GHz})$ & 30 & $h_{\text {nailr } 01}(\mathrm{~mm})$ & 1.012 & 0.989 \\
\hline$f_{02}(\mathrm{GHz})$ & 30 & $h_{\text {nailr } 02}(\mathrm{~mm})$ & 1.181 & 1.188 \\
\hline
\end{tabular}

As we did before, a new design curve (using again $A N$ SYS HFSS for the full-wave electromagnetic analysis of the structure in Fig. 13) that relates the values of $k$ that can be achieved with different separation $\left(s_{\text {gap }}\right)$ between the coupled resonators was obtained. These results (included in Fig. 14) have been used to select initial values for $s_{\text {gap } 12}=3.715 \mathrm{~mm}$ and $s_{\text {gap } 23}=3.892 \mathrm{~mm}$, which are able to provide, respectively, the requested ideal values for $k_{12}$ and $k_{23}$ of 0.0152 and 0.0117 .

Again, due to the loading effect of the physical coupling on each adjacent cavity, their corresponding resonant frequencies are slightly detuned with regard to the center frequency of the bandpass response. In order to compensate this effect, a new value for the height of the shortened nails in the second and third cavities are obtained $\left(h_{\text {nailr } 02}=1.181 \mathrm{~mm}\right)$. In order to find such value, a design curve relating the resonant frequency of the two coupled cavities (Fig. 13) with regard to $h_{\text {nailr } 02}$ has been obtained. It is again very similar to the one included in Fig. 7, but its linear approximation has now a slope $m=-$ $21.0 \mathrm{GHz} / \mathrm{mm}$.

\section{Filter Optimization}

A complete view of the proposed combline filter in gap waveguide technology can be seen in Fig. 15, and the initial values for all involved geometrical variables (obtained with the method just described before) are collected in Table I. The full-wave simulated results of the initially designed structure are shown in Fig. 16, where the ideal response of the equivalent circuit representation (with the optimum values of the electrical parameters summarized in Table I) is also included.

As can be seen in Fig. 16, the initial design of the proposed filter does not provide good enough results, so an optimization 

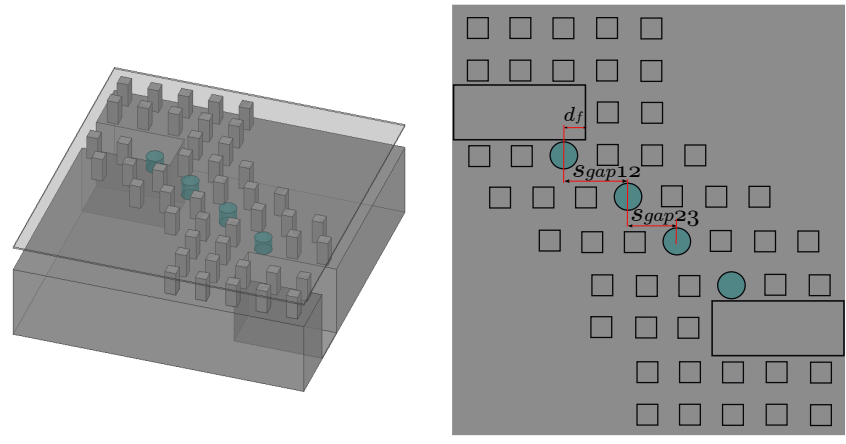

Fig. 15. 3D and 2D views of the proposed coaxial filter with shortened nails in gap waveguide technology.

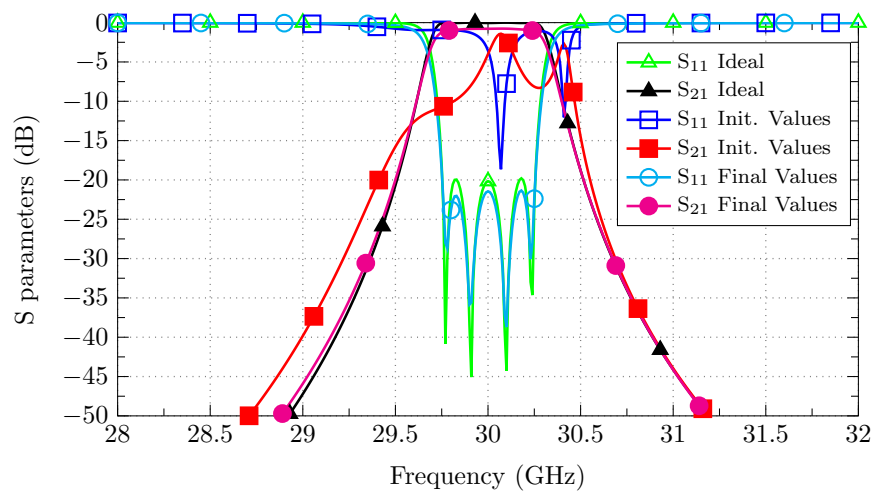

Fig. 16. Ideal and full-wave simulated responses (S-parameters) of the considered filter.

procedure of all involved geometrical variables should be performed in order to get results much closer to the ideal response. This optimization stage could be directly done using the employed full-wave electromagnetic simulator, but the involved computational effort (due to the intensive use of the employed accurate software tool) would be unaffordable.

In order to enhance this last optimization stage of the design procedure, we proposed in this work to use the Aggressive Space Mapping (ASM) technique, originally proposed by Prof. Bandler et al. in [26], and updated here (as detailed next) to make an efficient use of the equivalent circuit model based on coupled resonators (see Fig. 8). There are other alternative powerful approaches, that using model estimation and extraction methods, get the tuning of practical lossy microwave filters [34], [35] and [36].

Any ASM method makes use of two variable (or parameter) spaces, where two different tools (or models, known as "coarse" and "fine") are used for performing simulations of the component to be designed. In the coarse model space, an efficient simulation tool provides results without enough accuracy, whereas a more accurate analysis method (with a higher computational cost) is employed in the fine model space. Then, an iterative mapping between both simulation spaces is performed, thus finally providing an accurate optimal solution with a few number of fine model simulations.

In our particular case, the coarse space is defined with the five electrical parameters used as input variables in the equivalent circuit of the filter (see Fig. 8), i.e., $\mathbf{x}_{c}=$
$\left[\begin{array}{lllll}Q_{e} & k_{12} & k_{23} & f_{01} & f_{02}\end{array}\right]^{T}$, and the corresponding coarse model response, denoted as $\mathbf{R}_{c}\left(\mathbf{x}_{c}\right)$, will be obtained with the previously employed tool (i.e., $A D S$ from Keysight). On the other hand, the fine space parameters are the physical dimensions of the proposed filter topology, i.e., $\mathbf{x}_{f}=$ $\left[\begin{array}{lllll}d_{f} & s_{\text {gap } 12} & s_{\text {gap } 23} & h_{\text {nailr } 01} & h_{\text {nailr } 02}\end{array}\right]^{T}$, and ANSYS HFSS tool will provide the fine model response, i.e., $\mathbf{R}_{f}\left(\mathbf{x}_{f}\right)$. In order to start the ASM algorithm, we need to know an optimal solution in the coarse model space, called $\mathbf{x}_{c}^{*}$, whose values are the ones for the electrical parameters in Table I, and a first approximated solution in the fine model space, labelled as $\mathbf{x}_{f}^{(1)}$, which is obtained as indicated in Sections IV-A and IV-B (i.e., the initial values for the physical dimensions collected in Table I).

Then, an accurate mapping between the fine and coarse spaces, defined as $\mathbf{x}_{c}=\mathbf{P}_{f c}\left(\mathbf{x}_{f}\right)$, must be established. For this purpose, the HFSS fine model response, $\mathbf{R}_{f}\left(\mathbf{x}_{f}\right)$, will be fully recovered through an optimization process performed with the $A D S$ software tool, which provides the values of the electrical parameters of the equivalent circuit, i.e., the corresponding $\mathbf{x}_{c}$.

Making use of the cited mapping procedure, we can easily obtain the first set of electrical parameters, i.e., $\mathbf{x}_{c}^{(1)}$, that provides the HFSS response shown in Fig. 16 for the initial values of the physical variables; and then compute the deviation between this solution and the optimal one in the coarse space, i.e., $\Delta \mathbf{x}_{c}^{(1)}=\mathbf{P}_{f c}\left(\mathbf{x}_{f}^{(1)}\right)-\mathbf{x}_{c}^{*}$.

Now, the iterative part of the ASM algorithm can be applied, thus solving the next equation for the $j$-th iteration (with $j=1,2, \ldots)$ :

$$
\mathbf{B}^{(j)} \Delta \mathbf{x}_{f}^{(j)}=-\Delta \mathbf{x}_{c}^{(j)}
$$

and evaluating the new solution in the fine space as follows:

$$
\mathbf{x}_{f}^{(j+1)}=\mathbf{x}_{f}^{(j)}+\Delta \mathbf{x}_{f}^{(j)}
$$

In (10), $\mathbf{B}^{(j)}$ represents the approximation to the Jacobian (or mapping) matrix of the ASM algorithm at iteration $\mathrm{j}$; that is updated by the classical Broyden formula, [37]:

$$
\mathbf{B}^{(j+1)}=\mathbf{B}^{(j)}+\frac{\Delta \mathbf{x}_{c}^{(j+1)} \Delta \mathbf{x}_{f}^{(j)^{T}}}{\Delta \mathbf{x}_{f}^{(j)^{T}} \Delta \mathbf{x}_{f}^{(j)}}
$$

Obviously, the mapping matrix must be initialized in the first iteration, i.e., $\mathbf{B}^{(1)}$, and this choice is key for the fastest and robust evolution of the ASM algorithm. In this work, we have used for $\mathbf{B}^{(1)}$ a diagonal matrix, whose meaningful elements are obtained as the derivatives of the corresponding design curves generated before (i.e., the ones included in Figs. 12 and 14), or taking directly the values of the slopes indicated in Sections IV-A and IV-B for the linear approximations of design curves similar to the one in Fig. 7.

The previous iterative process is ended when the fine model response at $j$-th iteration, $\mathbf{R}_{f}\left(\mathbf{x}_{f}^{(j)}\right)$, is close enough to the ideal one, i.e., $\mathbf{R}_{c}\left(\mathbf{x}_{c}^{*}\right)$. Thanks to the proposed initialization of the mapping matrix, in our filter example, we have just needed five iterations to reach the optimal solution in the fine model space (see final values in Table I). The full-wave simulated 


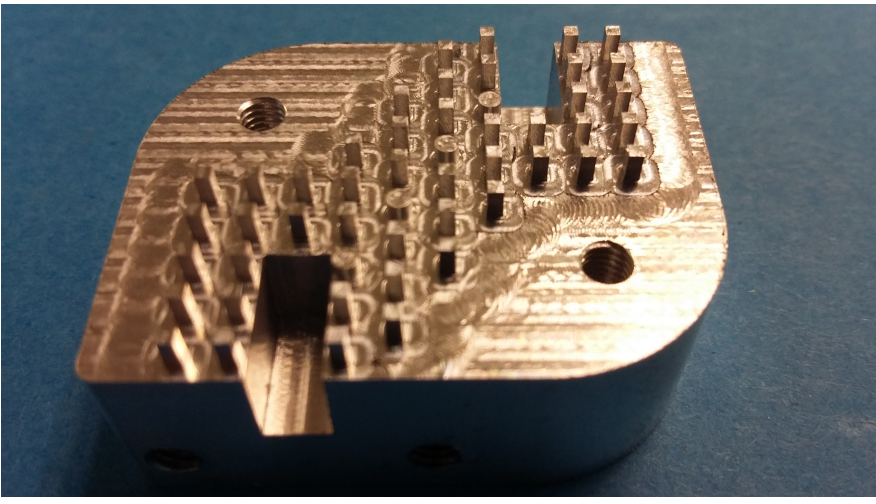

Fig. 17. Filter base piece, with periodic bed of nails, shortened cylindrical resonant nails, and input/output groove gap waveguides.

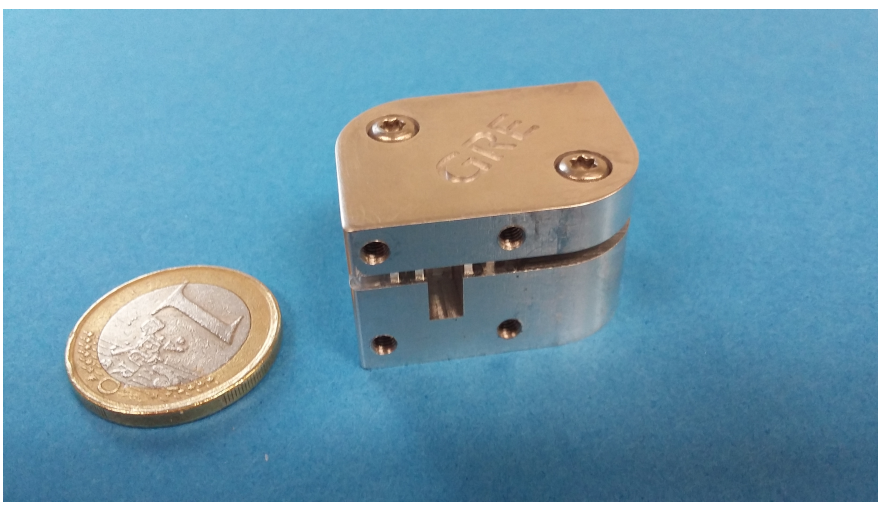

Fig. 18. Top metal cover of the filter fixed on top of the main body (base piece).

results of the designed combline filter, using ANSYS HFSS, is successfully compared to the ideal filter response in Fig. 16.

It is worth recalling that at each iteration of the ASM algorithm, the main computational cost is related to the corresponding full-wave HFSS simulation, being practically negligible the time effort requested by the optimization of the filter equivalent circuit performed with $A D S$. If the optimization process had been directly performed with the electromagnetic simulator, the number of requested iterations (and, therefore, of accurate simulations) would have been much larger than five, thus making the design process of the proposed filter rather inefficient and less robust.

\section{EXPERIMENTAL RESULTS}

The designed combline filter has been manufactured in gap waveguide technology, using a standard milling process with aluminum as base material. As it can be seen in Fig. 17, the supporting bed of nails, together with the excitation groove gap waveguides (GGWs) and the shortened resonant nails, are included in the same base piece (or main body of the filter). Then, a metal cover is fixed on top of the previous structure (see Fig. 18), ideally providing a perfect contact with the periodic bed of nails.

In Fig. 19 we show the measured results of the manufactured prototype, where it can be noticed a frequency shift of $210 \mathrm{MHz}$ with regard to the simulated filter response,

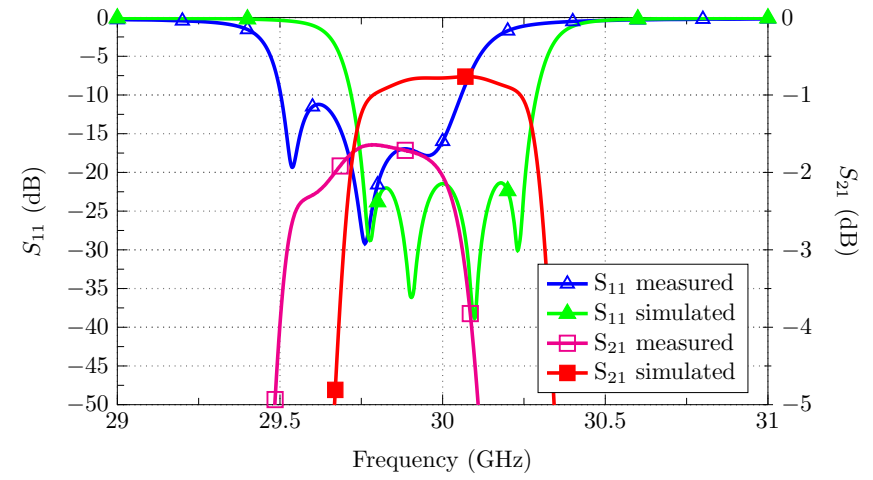

Fig. 19. Measured and simulated responses (S-parameters) of the manufactured filter prototype.

and in-band return losses below the ideal requirement of 20 $\mathrm{dB}$. After performing an inspection of the main filter body with a metrology equipment, we detected an increment of 15 $\mu \mathrm{m}$ in the height of the shortened nails with regard to the final design values, which explains the observed frequency shift. Furthermore, the corners of the input/output GGWs were not finished well enough, thus changing the value of the achieved external quality factor (affecting the measured in-band return loss level). In order to verify this, a new simulation taking into account the manufacturing errors has been done. Fig. 20 shows the new simulation results compared with measurements. It can be noted the agreement between the two responses of Fig. 20. These manufacturing errors could be taken into account in the filter design stage, thus alleviating its undesired effects with a second fabrication round. However, in order to avoid the manufacturing of a new filter prototype, we have tried to enhance the previous results for the in-band return losses by adding input/output matching networks. Taking advantage of available sections of standard WR 28 waveguide of $3 \mathrm{~cm}$ fixed length, each matching network is composed of one piece of this waveguide length located between two reactive irises, which have been properly designed for matching purposes. A view of the manufactured pieces for the designed irises, as well as of the final test set-up (including the filter with the input/output matching networks), can be seen in Figs. 21 and 22, respectively.In order to avoid the use of these input/output matching networks, embedded screws located on top of the coaxial cavities and coupling apertures of the filter could have been used too. After properly fixing all these tuning screws, the measured response can be shifted in frequency to the desired nominal value, and the related return loss response substantially enhanced.

The measured results of the manufactured filter prototype, including now the designed input/output matching networks, is shown in Fig. 23, where it can be appreciated that in-band results have been improved (with regard to the experimental data of Fig. 19). In particular, a return loss level higher than $16.6 \mathrm{~dB}$ has been recovered in the frequency range between $29.535 \mathrm{GHz}$ and $30.015 \mathrm{GHz}$, thus providing a useful bandwidth of $480 \mathrm{MHz}$ (rather close to the $500 \mathrm{MHz}$ specification of the ideal filter response). A minimum value of $1.607 \mathrm{~dB}$ has been measured for the in-band insertion losses 


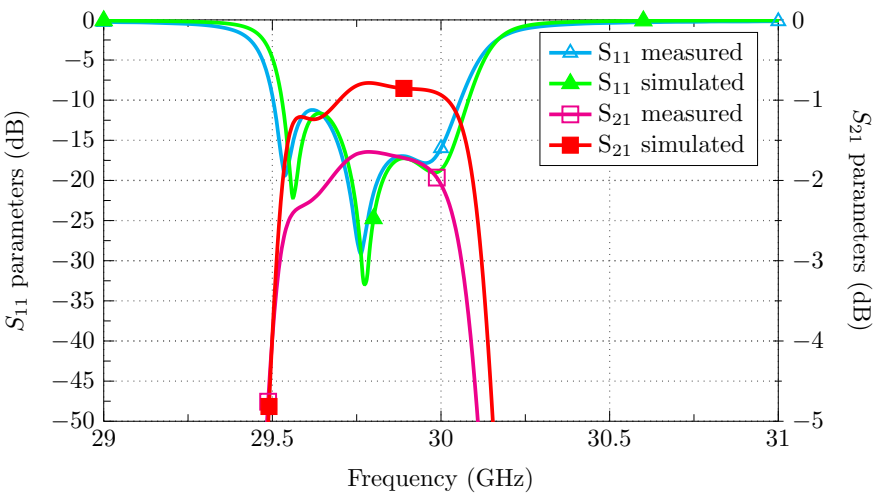

Fig. 20. Measured and simulated with manufactured errors responses (Sparameters) of the filter prototype.

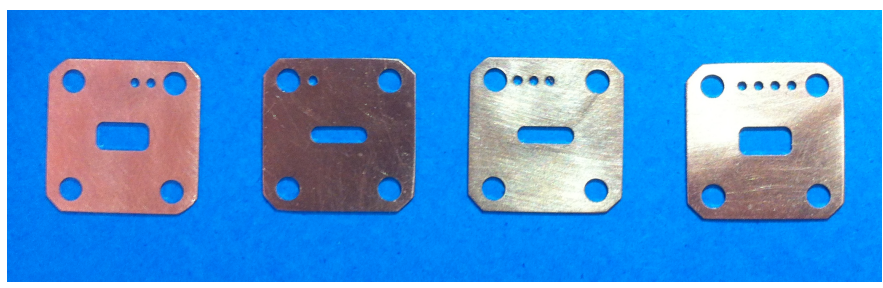

Fig. 21. View of the manufactured irises of the designed matching networks.

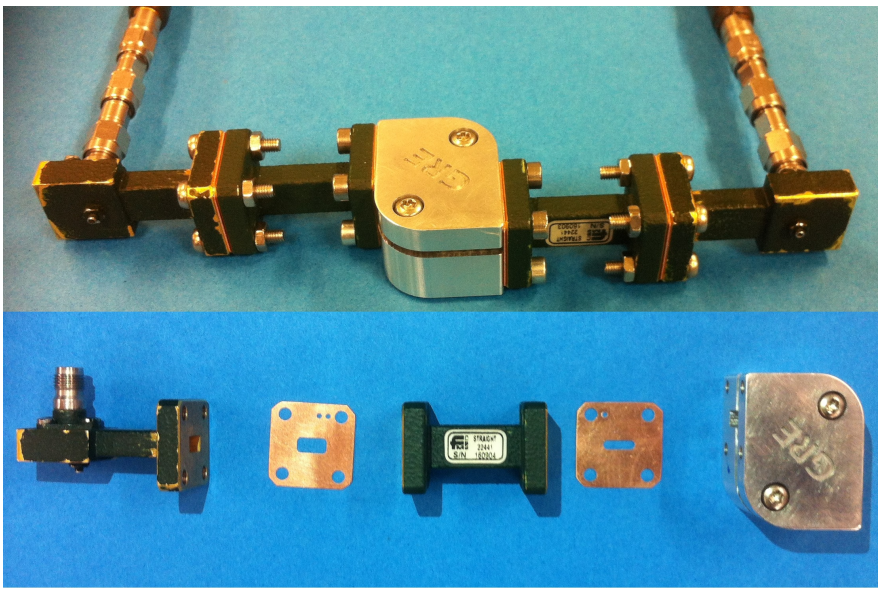

Fig. 22. Complete view of the test set-up for measuring the manufactured filter with input/output matching networks.

of the filter (including the matching networks), whereas the simulated value with ANSYS HFSS for the designed filter (without matching networks and considering aluminum as material) was just $0.76 \mathrm{~dB}$.

\section{CONCLUSION}

A compact topology for implementing combline filters in gap waveguide technology, providing bandpass responses at high frequencies, has been successfully proposed. For this purpose, we have employed shortened cylindrical nails as resonators, which are fully integrated in a periodic structure (bed of square nails) with a stopband behaviour. The resonance behaviour of the considered resonators has been studied and properly modeled with an equivalent circuit. Effective coupling mechanisms between adjacent resonators, as well as for the

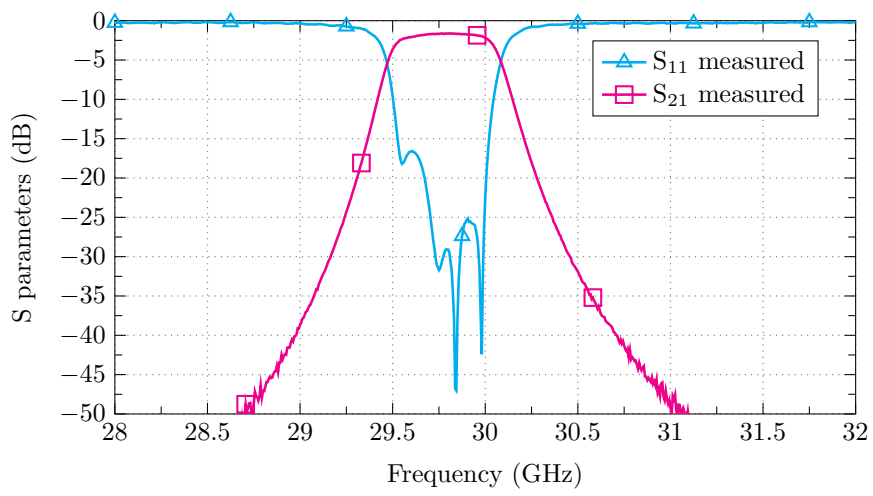

Fig. 23. Measured results (S-parameters) of the manufactured filter prototype including the input/output matching networks.

input and output excitation of the filter with GGWs, have been detailed; including design curves relating the physical parameters of the filter topology with electrical parameters of an equivalent circuit representation. A more efficient procedure for optimizing the physical dimensions of the proposed filter topology, making use of an updated version of the ASM technique, has been also derived and illustrated with a practical design example. For validation purposes, a prototype of a designed combline filter (with a bandpass response of $1.7 \%$ relative bandwidth at $30 \mathrm{GHz}$ ) has been manufactured and measured. The proposed filters can be easily embedded, together with other components, within a hosting structure based on a bed of nails, thus making feasible the practical realization of integrated subsystems in gap waveguide technology.

\section{REFERENCES}

[1] R. E. Collin, Foundations for Microwave Engineering. New York: McGraw-Hill International Editions, 1992.

[2] P. S. Kildal, "Three metamaterial-based gap waveguides between parallel metal plates for mm/submm waves," in Proc. 3rd Eur. Conf. Antennas Propag. (EuCAP), March 2009, pp. 28-32.

[3] P. S. Kildal, E. Alfonso, A. Valero-Nogueira, and E. Rajo-Iglesias, "Local metamaterial-based waveguides in gaps between parallel metal plates," IEEE Antennas Wireless Propag. Lett., vol. 8, pp. 84-87, 2009.

[4] E. Alfonso, M. Baquero, P. S. Kildal, A. Valero-Nogueira, E. RajoIglesias, and J. I. Herranz, "Design of microwave circuits in ridge-gap waveguide technology," in IEEE MTT-S Int. Microw. Symp. Dig., May 2010, pp. 1544-1547.

[5] A. Berenguer, V. Fusco, D. E. Zelenchuk, D. Sánchez-Escuderos, M. Baquero-Escudero, and V. E. Boria-Esbert, "Propagation characteristics of groove gap waveguide below and above cutoff," IEEE Trans. Microw. Theory and Techn., vol. 64, no. 1, pp. 27-36, Jan. 2016.

[6] A. U. Zaman, T. Vukusic, M. Alexanderson, and P. S. Kildal, "Design of a simple transition from microstrip to ridge gap waveguide suited for mmic and antenna integration," IEEE Antennas Wireless Propag. Lett., vol. 12, pp. 1558-1561, 2013.

[7] A. U. Zaman, V. Vassilev, P. S. Kildal, and H. Zirath, "Millimeter wave e-plane transition from waveguide to microstrip line with large substrate size related to mmic integration," IEEE Microw. Compon. Lett., vol. 26, no. 7, pp. 481-483, July 2016.

[8] A. A. Brazález, J. Flygare, J. Yang, V. Vassilev, M. Baquero-Escudero, and P. S. Kildal, "Design of $f$-band transition from microstrip to ridge gap waveguide including monte carlo assembly tolerance analysis," IEEE Trans. Microw. Theory and Techn., vol. 64, no. 4, pp. 1245-1254, April 2016.

[9] S. I. Shams and A. A. Kishk, "Wideband coaxial to ridge gap waveguide transition," IEEE Trans. Microw. Theory and Techn., vol. 64, no. 12, pp. 4117-4125, Dec. 2016. 
[10] U. Nandi, A. U. Zaman, A. Vosoogh, and J. Yang, "Novel millimeter wave transition from microstrip line to groove gap waveguide for mmic packaging and antenna integration," IEEE Microw. Compon. Lett., vol. 27, no. 8, pp. 691-693, Aug. 2017.

[11] A. Farahbakhsh, D. Zarifi, and A. U. Zaman, "60 GHz groove gap waveguide based wideband H-Plane power dividers and transitions: For use in high-gain slot array antenna," IEEE Trans. Microw. Theory Techn., vol. 65 , no. 11 , pp. 4111-4121, Nov. 2017.

[12] A. Tamayo-Domínguez, J. M. Fernández-González, and M. Sierra-Pérez, "Groove gap waveguide in 3-D printed technology for low loss, weight, and cost distribution networks," IEEE Trans. Microw. Theory Techn., vol. 65 , no. 11, pp. 4138-4147, Nov. 2017.

[13] A. del Olmo-Olmeda, M. Baquero-Escudero, V. E. Boria-Esbert, A. Valero-Nogueira, and A. J. Berenguer-Verdú, "A novel band-pass filter topology for millimeter-wave applications based on the groove gap waveguide," in IEEE MTT-S Int. Microw. Symp. Dig.), June 2013, pp. $1-4$.

[14] E. A. Alós, A. U. Zaman, and P. S. Kildal, "Ka-band gap waveguide coupled-resonator filter for radio link diplexer application," IEEE Trans. Compon. Packag. Manuf. Technol., vol. 3, no. 5, pp. 870-879, May 2013.

[15] A. Berenguer, M. Baquero-Escudero, D. Sanchez-Escuderos, B. Bernardo-Clemente, and V. E. Boria-Esbert, "Low insertion loss 61 $\mathrm{GHz}$ narrow-band filter implemented with groove gap waveguides," in Proc. 44th Eur. Microw. Conf., Oct. 2014, pp. 191-194.

[16] B. Ahmadi and A. Banai, "Direct coupled resonator filters realized by gap waveguide technology," IEEE Trans. Microw. Theory and Techn., vol. 63, no. 10, pp. 3445-3452, Oct. 2015.

[17] D. Sun and J. Xu, "A novel iris waveguide bandpass filter using air gapped waveguide technology," IEEE Microw. Compon. Lett., vol. 26 , no. 7, pp. 475-477, July 2016.

[18] M. S. Sorkherizi and A. A. Kishk, "Completely tuned coupled cavity filters in defected bed of nails cavity," IEEE Trans. Compon. Packag. Manuf. Technol., vol. 6, no. 12, pp. 1865-1872, Dec 2016.

[19] A. Vosoogh, M. S. Sorkherizi, A. U. Zaman, J. Yang, and A. A. Kishk, "An integrated ka-band diplexer-antenna array module based on gap waveguide technology with simple mechanical assembly and no electrical contact requirements," IEEE Trans. Microw. Theory and Techn., vol. 66, no. 2, pp. 962-972, Feb 2018.

[20] A. J. Sáez, A. Valero-Nogueira, J. I. Herranz, and V. M. Rodrigo, "Ring resonances in groove gap waveguides with application to slot array antennas," in Proc. IEEE Int. Symp. Antennas . Propag. USNC/URSI National Radio Science Meeting, July 2015, pp. 260-261.

[21] A. J. Sáez, A. Valero-Nogueira, J. I. Herranz, and B. Bernardo, "Singlelayer cavity-backed slot array fed by groove gap waveguide," IEEE Antennas Wireless Propag. Lett., vol. 15, pp. 1402-1405, 2016.

[22] M. Ferrando-Rocher, A. Valero-Nogueira, J. I. Herranz-Herruzo, and A. Berenguer, "V-band single-layer slot array fed by ridge gap waveguide," in Proc. IEEE Int. Symp. Antennas .Propag. (APSURSI), June 2016, pp. 389-390.

[23] M. Ferrando-Rocher, A. Valero-Nogueira, and J. I. Herranz-Herruzo, "New feeding network topologies for high-gain single-layer slot array antennas using gap waveguide concept," in Proc. 11th Eur. Conf. Antennas Propag. (EuCAP), March 2017, pp. 1654-1657.

[24] H.-W. Yao, K. A. Zaki, A. E. Atia, and R. Hershtig, "Full wave modeling of conducting posts in rectangular waveguides and its applications to slot coupled combline filters," IEEE Trans. Microw. Theory and Techn., vol. 43, no. 12, pp. 2824-2830, Dec 1995.

[25] R. Cameron, C. M. Kudsia, and R. Mansour, Microwave Filters for Communication Systems, 2nd edition. John Wiley \& Sons, 2018.

[26] J. W. Bandler, R. M. Biernacki, S. H. Chen, R. H. Hemmers, and K. Madsen, "Electromagnetic optimization exploiting aggressive space mapping," IEEE Trans. Microw. Theory and Techn., vol. 43, no. 12, pp. 2874-2882, Dec. 1995.

[27] J.-H. Hong, Microstrip Filters for RF/Microwave Applications, 2nd edition. John Wiley \& Sons, 2011.

[28] A. Valero-Nogueira, E. Alfonso, J. I. Herranz, and P. S. Kildal, "Experimental demonstration of local quasi-tem gap modes in single-hard-wall waveguides," IEEE Microw. Compon. Lett., vol. 19, no. 9, pp. 536-538, Sept. 2009.

[29] E. Rajo-Iglesias and P. S. Kildal, "Numerical studies of bandwidth of parallel-plate cut-off realised by a bed of nails, corrugations and mushroom-type electromagnetic bandgap for use in gap waveguides," IET Microw., Antennas Propag., vol. 5, no. 3, pp. 282-289, Feb. 2011.

[30] S. I. Shamseldin, "Analysis and design of microwave devices based on ridge gap waveguide technology," Ph.D. dissertation, Concordia University Montréal, Quebec, Canada, 82016.
[31] N. Marcuvitz, Waveguide Handbook. New York: McGraw-Hill, 1955, sec. 4.5 .

[32] B. C. Wadell, Transmission Line Design Handbook. Norwood: Artech House, Inc. , 1991

[33] G. Matthaei, L. Young, and E. Jones, Microwave Filters, ImpedanceMatching Networks, and Coupling Structures. Norood MA: Artech House, 1980.

[34] M. Kahrizi, S. Safavi-Naeini, S. K. Chaudhuri, and R. Sabry, "Computer diagnosis and tuning of $\mathrm{rf}$ and microwave filters using model-based parameter estimation," IEEE Trans. Circuits Syst. I: Fundam. Theory Appl., vol. 49, no. 9, pp. 1263-1270, Sept 2002.

[35] M. Meng and K. Wu, "An analytical approach to computer-aided diagnosis and tuning of lossy microwave coupled resonator filters," IEEE Trans. Microw. Theory and Techn., vol. 57, no. 12, pp. 3188-3195, Dec 2009.

[36] M. Esmaeili and A. Borji, "Diagnosis and tuning of multiple coupled resonator filters," in Proc. 18th Iranian Conf. Elect. Eng., May 2010, pp. 124-129.

[37] C. G. Broyden, "Electromagnetic optimization exploiting aggressive space mapping," Math. Comp., vol. 19, pp. 577-593, 1965.

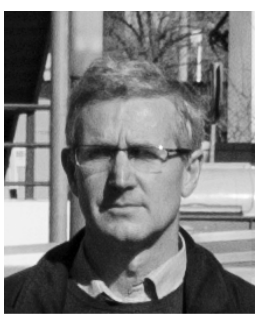

Mariano Baquero-Escudero (S87-M90) was born in Murcia, Spain, on January 11, 1962. He received the degree in telecommunications engineering from the Polytechnic University of Catalonia (UPC), Barcelona, Spain, in 1986 and the Ph.D. degree from the Polytechnic University of Valencia (UPV), Valencia, Spain, in 1994. He became a Member (M) of IEEE in 1987. He was with the Antennas, Microwave and Radar Group, UPC, from 1986 to 1988 , where he worked on the development of a cylindrical near-field facility to measure a 3-D radar antenna in CESELSA. Since 1989, he has been with the UPV where he became a Full Professor in 2003. During 1995, he held a postdoctoral grant at the Joint Research Centre, European Commission, Ispra, Italy, where he developed high-resolution algorithms for radar applications. From April 1996 to February 1998, he was a Vice-Dean of the Telecommunications Engineering School of Valencia. He is currently with the Communications Department and into the Institute of Telecommunications and Multimedia Application of the Polytechnic University of Valencia. His main research interests include microwave circuit and antenna analysis, design and measurement.

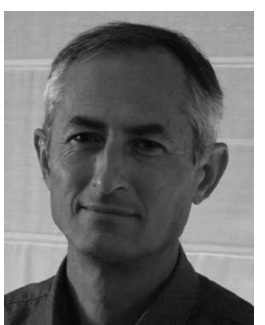

Alejandro Valero-Nogueira (S92-M97-SM09) was born in Madrid, Spain, on July 19, 1965. He received the Ingeniero de Telecomunicacion degree from the Universidad Politecnica de Madrid, Madrid, in 1991 and the Ph.D. degree in Telecommunication from the Universitat Polit'ecnica de Val'encia, Val'encia, Spain, in 1997. In 1992, he joined the Departamento de Comunicaciones, Universitat Polit'ecnica de Val'encia, where he is currently a Full Professor. His current research interests include computational electromagnetics, waveguide slot arrays, gap waveguides, and new antenna architectures for SATCOM terminals. 


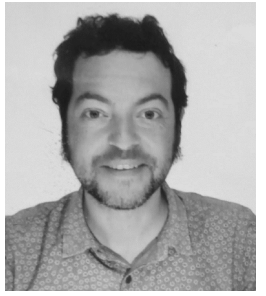

Miguel Ferrando-Rocher (S15) was born in Alcoy, Spain, in 1985. He received the M.Sc. degree in telecommunication engineering from the Universitat Polit'ecnica de Val'encia, Spain, in 2012, and he is currently working towards the Ph.D. degree in telecommunication engineering. He received a Erasmus grant to study in Ghent University (Belgium) in 2010. He joined as a researcher in the Complex Radiation Systems team of IETR in Rennes (France) in 2012, where he worked on Reflectarray Antennas for Satellite Applications in collaboration with Thales Alenia Space. Since 2013, he works in the Electromagnetic Radiation Group of the Institute of Telecommunications and Multimedia Applications of the UPV. He visited Chalmers University of Technology (Sweden) as Guest Researcher in 2016. His current research interests include satellite communications on-the-move, high-gain antennas and arrays, gap waveguide technology, and mm-wave components. Ferrando-Rocher was a recipient of the URSI Conference Best Student Paper Award in 2017 and the Jury prize and the audience prize to the best oral presentation in the II Meeting of $\mathrm{PhD}$ Students at UPV (Spain)

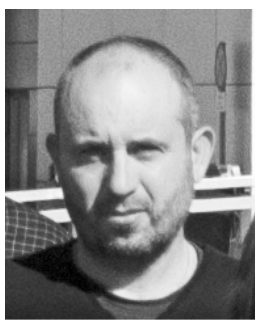

Bernardo Bernardo-Clemente was born in Valencia, Spain, on May 8, 1972. He received the degree in telecommunication engineering from Univeritat Politcnica de Valncia in 2003. He is currently working toward the $\mathrm{Ph}$. D. degree in telecommunication. He has been with the Institute of Telecommunicactions and Multimedia Applications (ITEAM) since 2005. His main research interests include antenna measurement, antenna fabrication, near to far field transformation.

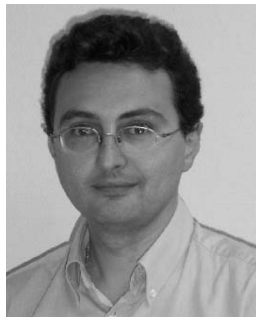

Vicente E. Boria-Esbert (S91A99SM02F18) was born in Valencia, Spain, in 1970. He received the Ingeniero de Telecomunicacin degree (Hons.) and Doctor Ingeniero de Telecomunicacin degree from the Universidad Politcnica de Valencia, Valencia, Spain, in 1993 and 1997, respectively. In 1993, he joined the Departamento de Comunicaciones, Universidad Politcnica de Valencia, where he has been a Full Professor since 2003. In 1995 and 1996, he held a Spanish Trainee position with the European Space Research and Technology Centre, European Space Agency, Noordwijk, The Netherlands, where he was involved in the area of electromagnetic analysis and design of passive waveguide devices. $\mathrm{He}$ has authored or co-authored 10 chapters in technical textbooks, 160 papers in refereed international technical journals, and over 200 papers in international conference proceedings. His current research interests include the analysis and automated design of passive components, left-handed and periodic structures, as well as on the simulation and measurement of high power effects in passive waveguide systems. Dr. Boria has been a member of the IEEE Microwave Theory and Techniques Society (IEEE MTT-S) and the IEEE Antennas and Propagation Society since 1992. He is a member of the Editorial Boards of the IEEE TRANSACTIONS ON MICROWAVE THEORY AND TECHNIQUES, IEEE MICROWAVE AND WIRELESS COMPONENTS LETTERS, Proceedings of the IET (Microwaves, Antennas, and Propagation), and IET Electronics Letters and Radio Science. He currently serves as an Associate Editor of IEEE MICROWAVE AND WIRELESS COMPONENTS LETTERS and IET Electronics Letters. He is also a member of the Technical Committees of the IEEEMTT- S International Microwave Symposium Conference and the European Microwave Conference. 